\title{
High-energy neutrinos and gamma rays from the AGN-driven wind in NGC 1068
}

\author{
Susumu Inoue, ${ }^{a, b, *}$ Matteo Cerruti, ${ }^{c}$ Kohta Murase ${ }^{d, e}$ and Ruoyu Liu ${ }^{f}$ \\ ${ }^{a}$ Bunkyo University, Koshigaya, Saitama, Japan \\ ${ }^{b}$ RIKEN, Wako, Saitama, Japan \\ ${ }^{c}$ AstroParticle et Cosmologie, Paris, France \\ ${ }^{d}$ Penn State University, University Park, USA \\ ${ }^{e}$ Yukawa Institute of Theoretical Physics, Kyoto University, Kyoto, Japan \\ ${ }^{f}$ Nanjing University, Nanjing, China \\ E-mail: susumu.inoue@riken.jp
}

\begin{abstract}
Various observations are revealing the widespread occurrence of fast and powerful winds in active galactic nuclei (AGN) that are distinct from relativistic jets, likely launched from accretion disks. Such winds can harbor collisionless shocks at different locations that may induce acceleration of protons and electrons and consequent nonthermal emission. We focus on the inner regions of the winds, where interactions of accelerated protons with the AGN radiation field and/or ambient gas clouds can induce emission of high-energy neutrinos and gamma rays. In particular, we address the case of NGC 1068, a nearby Seyfert galaxy bearing a prominent wind, which is a known source of $\mathrm{GeV}$ gamma rays as well as a tentative source of sub-PeV neutrinos. Considering $p \gamma, p p$, as well as all relevant electromagnetic processes in such regions, we model the multi-messenger emission of NGC 1068. The relatively soft neutrino spectrum indicated by IceCube may favor a predominantly $p p$ interpretation in which high-energy protons interact with gas that has densities typical of broad line region clouds. The accompanying cascade emission modulated by $\gamma \gamma$ absorption in the AGN radiation field can account for the observed $\mathrm{GeV}$ emission under certain conditions.
\end{abstract}

$37^{\text {th }}$ International Cosmic Ray Conference (ICRC 2021)

July 12th - 23rd, 2021

Online - Berlin, Germany

\footnotetext{
*Presenter
} 


\section{Introduction}

Powerful, highly collimated jets of predominantly non-thermal plasma with ultra-relativistic outflow velocities are known to be produced in less than $10 \%$ of all active galactic nuclei (AGN) in the present Universe [1]. On the other hand, there is widespread evidence that AGN can more commonly eject moderately collimated winds of thermal plasma, with outflow velocities from a few $100 \mathrm{kms}^{-1}$ up to mildly relativistic values of $\sim 0.3 c$, primarily observed as blue-shifted absorption features due to ionized metals at UV and X-ray energies [2]. They are seen in at least $\sim 40 \%$ of all nearby AGN, of both radio-loud and radio-quiet types (i.e. with or without strong jets). The winds are inferred to be generated on sub-pc scales, and their estimated kinetic power can reach a fair fraction of the AGN bolometric luminosity.

Such AGN-driven winds may be launched from accretion disks by a variety of mechanisms involving thermal, radiative and magnetic processes [2]. They may play a crucial role in collimating relativistic jets in radio-loud AGN [1]. Furthermore, they may be the primary agents by which supermassive black holes provide mechanical and/or thermal feedback onto their host galaxies, potentially leading to the observed black hole scaling relations and/or the quenching of star formation in massive galaxies [4]. Recent observations of fast and massive outflows of atomic and molecular material from AGN may be manifestations of such effects, caused by the winds propagating to kpc scales and interacting strongly with the gas in their host galaxies [3].

Some recent theoretical studies have addressed the interaction of AGN-driven winds with the ambient gas in the host galaxy and/or halo, with attention to the physics of the resulting external shocks and its observational consequences in nonthermal emission [5-8]. In this regard, a particularly interesting object is NGC 1068, a nearby (distance $\sim 14 \mathrm{Mpc}$ ), type-2 Seyfert galaxy that is known to possess a conspicuous AGN-driven wind $[3,9,10]$. The $\mathrm{GeV}$ gamma rays detected from the object was initially suggested to be of starburst origin [11], but its nature remains open as this interpretation was called into question through detailed multiwavelength modeling $[12,13]$. One suggestion is $p p \pi^{0}$ gamma rays induced by protons accelerated in the AGN wind external shock of NGC 1068 [7].

More recently, NGC 1068 has been shown to be a tentative source of neutrinos by IceCube [14], its position being coincident with the most significant point in the northern hemisphere in a full-sky can. A $2.9 \sigma$ excess over background expectations is also found at its position in a source catalog search. The On the other hand, upper limits in gamma rays at energies above $0.2 \mathrm{TeV}$ for NGC 1068 by MAGIC provide important constraints on the origin of such neutrinos [15]. They essentially rule out scenarios in which the neutrino production site is optically thin to the accompanying gamma rays, including that involving wind external shocks [7]. Some recent proposals instead invoke proton acceleration and neutrino production very close to the central black hole so that the associated gamma rays are signifcantly absorbed via $\gamma \gamma$ interactions with the AGN radiation field [16-18]. However, the latter would be strong enough down to the $\mathrm{MeV}$ range, in which case the observed $\mathrm{GeV}$ gamma rays must be attributed to some other process.

Here we propose an alternative picture where proton acceleration occurs in the inner regions of the AGN-driven wind in NGC 1068, not far from the nucleus. The protons subsequently induce neutrino emission via $p \gamma$ interactions with the AGN radiation field, and/or $p p$ interactions with dense, ambient gas clouds that constitute the so-called broad line region [19-21]. The accompanying 
electromagnetic cascade emission is modulated by $\gamma \gamma$ interactions with the AGN radiation field. The resultant multi-messenger emission may be broadly consistent with that observed from NGC 1068. More details on this work will be presented in an upcoming paper.

\section{Formulation}

Shocks that are conducive to particle acceleration can occur in the inner regions of AGN winds in different ways. Internal shocks can be caused by velocity inhomogeneities, which are in turn triggered by time-variable wind ejection. The latter is supported observationally by the variability seen in X-ray absorption features, as well as theoretically by numerical simulations of winds driven by accretion disks [2]. Alternatively, shocks can also arise due to interaction of the wind with ambient gas clouds that constitute the broad line region (BLR) on subparsec scales [19-21]. Here we simply assume that acceleration of protons can occur in such regions, and defer detailed discussions of how such shocks form to future studies.

Building on previous work $[22,23]$, we utilize a numerical code for modeling the multimessenger (photon and neutrino) emission resulting from a population of accelerated protons and electrons that are injected into a magnetized region and interact with ambient radiation fields and matter. The emission region is a sphere of radius $R$ with a randomly-oriented magnetic field of amplitude $B$, through which plasma is flowing with velocity $v$. It is permeated by an external radiation field that serves as target photons for $\gamma \gamma$ as well as $p \gamma$ interactions, with a spectrum characteristic of an accretion disk plus hot corona system around a black hole (BH) of mass $M_{\mathrm{BH}}$, total luminosity $L_{\mathrm{disk}}$, and fractional luminosity $f_{\text {cor }}$ for the corona [24], and intensity diluted according to the distance $z$ from the BH. It also contains gas with density $n_{g}$ as the target for $p p$ interactions, for which we follow the parameterization of Kelner et al. [25]. Protons are injected into the region with a power-law energy distribution with index $s$, an exponential cutoff at energy $E_{p \text {, max }}$, and total power $L_{p}$. (Injection of primary electrons is not considered in this work.) Subsequent $p \gamma$ and $p p$ interactions lead to production of secondary hadrons, leptons and photons, some which induce neutrinos. Primary and secondary charged particles also generate photons through synchrotron and inverse Compton radiation, of which a fraction also provide target photons for $\gamma \gamma$ and $p \gamma$ interactions. Electrons and positrons spawned by $\gamma \gamma$ interactions trigger pair cascades. The steady state distribution of all particles and the resulting multi-messenger emission are obtained self-consistently by solving the coupled kinetic equations that account for their radiative and adiabatic losses.

For concreteness, we choose $M_{\mathrm{BH}}=10^{8} M_{\odot}, L_{\text {disk }}=10^{44} \mathrm{erg} \mathrm{s}^{-1}$, and $f_{\text {cor }}=0.1$. Note that as the bulk velocity of AGN winds are at most mildly relativistic, Doppler beaming effects are weak, and the emission is expected to be quasi-isotropic.

\section{Results and discussion}

First we sought an interpretation based purely on $p \gamma$ processes, without including any $p p$ processes. Considering locations near the nucleus at $z=10^{14}-10^{15} \mathrm{~cm}(\sim 7-70$ gravitational radii $R_{g}$ ), $p \gamma$ interactions with the AGN radiation field can induce neutrino emission at very high energies, with luminosity in line with that indicated by IceCube for NGC 1068. The accompanying cascade 
emission is heavily attenuated by $\gamma \gamma$ interactions above $\epsilon_{\gamma} \sim 10-30 \mathrm{GeV}$ and emerges mostly below, potentially consistent with GeV-TeV observations of NGC 1068. However, the resulting neutrino spectrum peaks at $\epsilon_{v} \sim \mathrm{PeV}$, reflecting the peak of the AGN spectrum at a few tens of $\mathrm{eV}$, which seems too hard compared to the IceCube data where the excess appears in the range $\epsilon_{v} \sim 0.2-30$ $\mathrm{TeV}$.

Therefore, we extend the model to include $p p$ interactions, which can enhance the neutrino emission at lower energies. The parameters chosen are: $R=10^{14} \mathrm{~cm}\left(\sim 7 R_{g}\right), z=3 \times 10^{14} \mathrm{~cm}$ $\left(\sim 20 R_{g}\right), B=500 \mathrm{G}, n_{g}=10^{11} \mathrm{~cm}^{-3}, v=0.2 c, E_{p, \max }=10^{14} \mathrm{eV}, s=-0.5, L_{p}=10^{44} \mathrm{erg} \mathrm{s}^{-1}$. Fig. 1 compares the timescales for adiabatic expansion, acceleration, and energy loss processes of the protons, while Fig. 2 shows the resulting multi-messenger spectral energy distribution, which may provide a fairly reasonable account of the observational data for NGC 1068.

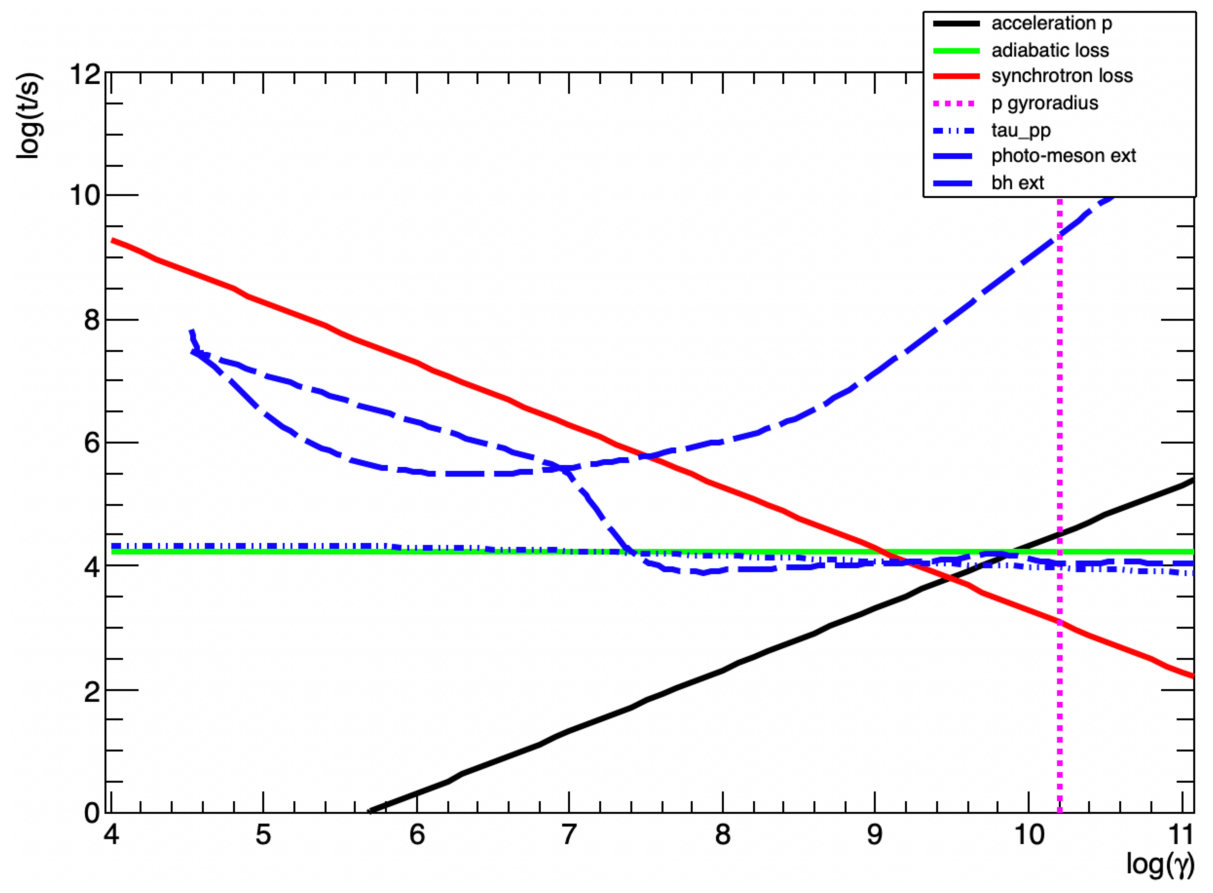

Figure 1: Comparison of timescales for adiabatic expansion (green solid), acceleration (black solid), proton synchrotron loss (red solid), photomeson loss (dashed), Bethe-Heitler loss (dot-dashed), proton-proton loss (double-dot-dashed). The acceleration timescale corresponds to the Bohm limit with shock velocity $v$. The vertical line indicates where the proton gyroradius equals $R$.

The gas density adopted here is a quite typical value inferred for BLR clouds, even though the BLR itself is not directly observable for NGC 1068 due to the high amount of obscuring material along the line of sight that is known for this object [19-21]. It is quite plausible that the wind with its broad opening angle interacts with some BLR clouds in its inner regions, resulting in the shocks that lead to proton acceleration as assumed above.

Fig. 1 provides a plausibility check on the chosen value of $E_{p \text {,max }}$. If the magnetic turbulence in the region is such that proton acceleration can proceed at the maximal, Bohm limit with shock velocity $v=0.2 c, E_{p, \max }$ can reach $>10^{18} \mathrm{eV}$ if limited by the dynamical timescale, or $>10^{16}$ $\mathrm{eV}$ if limited by photomeson losses. A much lower value as taken here can occur if the magnetic 


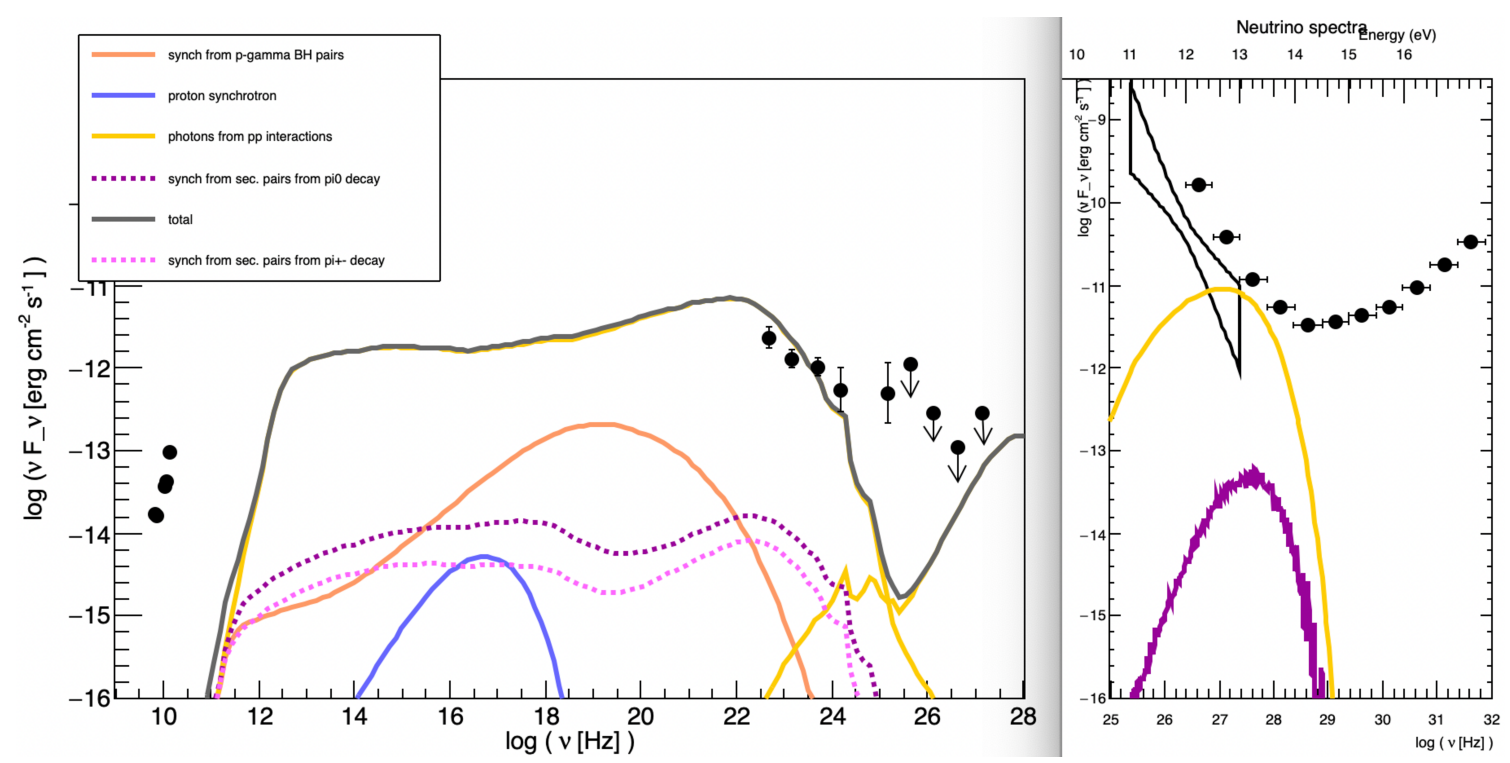

Figure 2: Multi-messenger spectral energy distribution for the model parameters discussed in the text, compared with observational data for NGC 1068. Left panel: Photon model curves for $\pi^{0}$ decay gamma rays and cascade emission due to $p p$ interactions (yellow solid), cascade emission due to $p \gamma$ Bethe-Heitler interactions (orange solid), cascade emission due to $p \gamma \pi^{0}$ and $\pi^{ \pm}$decay (purple and magenta dashed, respectively), protron synchrotron emission (blue solid). Data from Fermi-LAT [11] MAGIC [15] and ALMA [17]. Right panel: Neutrino model curves for $p p$ (yellow solid) and $p \gamma$ (purple solid). Data for NGC 1068 [14] and the 10-yr point-source sensitivity of IceCube.

turbulence is considerably weaker, e.g. as observational inferred for blazar jets [26], or if the shock velocity is much less.

The spectral index of the proton distribution below $E_{p \text {,max }}$ adopted here is appreciably harder than expectations from shock acceleration theory $(s \sim-2)$. This is required in order to keep the consequent cascade emission from overwhelming the observed $\mathrm{GeV}$ gamma rays. In principle, such a hard proton distribution may occur if one considers that the main region of $p p$ interactions is spatially separated from the main site of proton acceleration, from which the highest energy protons can escape more easily, analogous to situations expected for cosmic rays escaping from supernova remnants and interacting with molecular clouds in their vicinity [27]. Whether this can be plausibly realized in the current picture requires deeper investigation.

The neutrino flux and spectrum of the current model may still seem somewhat discrepant from what is indicated by IceCube observations of NGC 1068. Without invoking a nontrivial, hard proton distribution, an even higher neutrino flux that is compatible with the observed gamma rays may be achievable within the context of AGN-driven winds by relaxing the one-zone assumption for the entire multi-messenger emission. One may consider a primarily neutrino-emitting site with higher $\gamma \gamma$ optical depth, e.g. a location closer to the $\mathrm{BH}$, such that gamma rays associated with a higher neutrino flux is more strongly absorbed, similar to some of the aforementioned models [16-18]. In addition, a primarily gamma-ray emitting site with lower $\gamma \gamma$ optical depth can be envisaged, e.g. an external shock. Although such a model extension would imply more parameters, both regions are plausible within the context of an AGN-driven wind, long known to exist in NGC 1068. More 
detailed discussions will be presented in an upcoming paper.

\section{References}

[1] R. Blandford, D. Meier, A. Readhead, ARA\&A 57 (2019) 467.

[2] S. Laha, C. S. Reynolds, J. Reeves, G. Kriss, M. Guainazzi, R. Smith, S. Veilleux, D. Proga, Nat. Ast. 5 (2021) 13.

[3] S. Veilleux, R. Maiolino, A. D. Bolatto, S. Aalto, A\&Ap. Rev. 28 (2020) 2.

[4] C. M. Harrison, T. Costa, C. N. Tadhunter, A. Flütsch, D. Kakkad, M. Perna, G. Vietri, Nat. Ast. 2 (2021) 198.

[5] J. Nims, E. Quataert, C.-A. Faucher-Giguère, MNRAS 447 (2015) 3612.

[6] X. Wang, A. Loeb, MNRAS 453 (2015) 3612.

[7] A. Lamastra, F. Fiore, D. Guetta, L. A. Antonelli, S. Colafrancesco, N. Menci, S. Puccetti, A. Stamerra, L. Zappacosta, A\&A 596 (2016) A68.

[8] R.-Y. Liu, K. Murase, S. Inoue, C. Ge, X.-Y. Wang, ApJ 858 (2018) 9.

[9] G. Cecil, J. Bland, R. B. Tully, ApJ 355 (1990) 70.

[10] S. García-Burillo, F. Combes, C. Ramos Almeida, et al., A\&A 632 (2019) A61.

[11] M. Ackermann et al., ApJ 755 (2012) 164.

[12] T. M. Yoast-Hull, J. S. Gallagher, E. G. Zweibel, J. E. Everett, ApJ 780 (2014) 137.

[13] B. Eichmann, J. Becker Tjus, ApJ 821 (2016) 87.

[14] M. G. Aartsen et al., PRL 124 (2020) 051103.

[15] V. A. Acciari et al., ApJ 883 (2019) 135.

[16] K. Murase, S. S. Kimura, P. Mészáros, PRL 125 (2020) 011101.

[17] Y. Inoue, D. Khangulyan, A. Doi, ApJ 891 (2020) L33.

[18] L. A. Anchordoqui, J. F. Krizmanic, F. W. Stecker, PoS(ICRC2021)993. [arXiv:2102.12409].

[19] B. Peterson, Lecture Notes in Physics 693 (2006) 77.

[20] H. Netzer, ARA\&A 53 (2015) 365.

[21] C. Ramos Almeida, C. Ricci, Nat. Ast. 1 (2017) 679.

[22] M. Cerruti, A. Zech, C, Boisson, S. Inoue, MNRAS 448 (2015) 910. [arXiv: 1411 . 5968]. 
[23] M. Cerruti, M. Kreter, M. Petropoulou, A. Rudolph, F. Oikonomou et al., PoS(ICRC2021)979.

[24] M. Arrieta-Lobo, C. Boisson, A. Zech, FrASS 4 (2017) 56.

[25] S. R. Kelner, F. A. Aharonian, V. V. Bugayov, PRD 74 (2006) 034018. [arXiv: astro-ph/0606058].

[26] S. Inoue, F. Takahara, ApJ 463 (1996) 555.

[27] S. Gabici, F. A. Aharonian, ApJ 665 (2007) L131. [arXiv: 0705 . 3011]. 